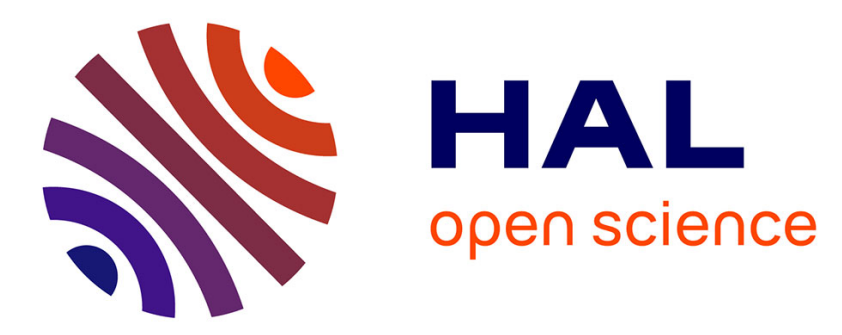

\title{
Interfacial electro-mechanical behaviour at rough surfaces
}

Chongpu Zhai, Dorian Hanaor, Gwénaëlle Proust, Laurence Brassart, Yixiang Gan

\section{- To cite this version:}

Chongpu Zhai, Dorian Hanaor, Gwénaëlle Proust, Laurence Brassart, Yixiang Gan. Interfacial electromechanical behaviour at rough surfaces. Extreme Mechanics Letters, 2016, 9 (3), pp.422-429. hal02307660

\section{HAL Id: hal-02307660 \\ https://hal.science/hal-02307660}

Submitted on 7 Oct 2019

HAL is a multi-disciplinary open access archive for the deposit and dissemination of scientific research documents, whether they are published or not. The documents may come from teaching and research institutions in France or abroad, or from public or private research centers.
L'archive ouverte pluridisciplinaire HAL, est destinée au dépôt et à la diffusion de documents scientifiques de niveau recherche, publiés ou non, émanant des établissements d'enseignement et de recherche français ou étrangers, des laboratoires publics ou privés. 


\title{
Interfacial electro-mechanical behaviour at rough surfaces
}

\author{
Chongpu Zhai ${ }^{\text {a }}$, Dorian Hanaor ${ }^{\text {a }}$, Gwénaëlle Proust ${ }^{\text {a }}$, Laurence Brassart ${ }^{\mathrm{b}}$, Yixiang Gan ${ }^{\text {a* }}$ \\ a School of Civil Engineering, The University of Sydney, NSW 2006, Australia. \\ ${ }^{\mathrm{b}}$ Department of Materials Science and Engineering, Monash University, VIC 3800, Australia. \\ * Corresponding author: yixang.gan@sydney.edu.au
}

\begin{abstract}
In a range of energy systems, interfacial characteristics at the finest length scales strongly impact overall system performance, including cycle life, electrical power loss, and storage capacity. In this letter, we experimentally investigate the influence of surface topology on interfacial electromechanical properties, including contact stiffness and electrical conductance at rough surfaces under varying compressive stresses. We consider different rough surfaces modified through polishing and/or sand blasting. The measured normal contact stiffness, obtained through nanoindentation employing a partial unloading method, is shown to exhibit power law scaling with normal pressure, with the exponent of this relationship closely correlated to the fractal dimension of the surfaces. The electrical contact resistance at interfaces, measured using a controlled current method, revealed that the measured resistance is affected by testing current, mechanical loading, and surface topology. At a constant applied current, the electrical resistance as a function of applied normal stress is found to follow a power law within a certain range, the exponent of which is closely linked to surface topology. The correlation between stress-dependent electrical contact and normal contact stiffness is discussed based on simple scaling arguments. This study provides a first-order investigation connecting interfacial mechanical and electrical behaviour, applicable to studies of multiple components in energy systems.
\end{abstract}

Keywords: Rough surfaces; contact stiffness; electrical contact resistance; electro-mechanical behaviour.

\section{Introduction}

Interfacial electro-mechanical behaviour is fundamental to indicators of energy system performance such as electrical power loss, cycle life, and storage capacity in lithium-ion batteries [1, 2], sodium-ion batteries [3], solid oxide fuel cells [4], photovoltaics [5] and thermoelectric systems [6]. Surface morphology plays an essential role in 
determining how contacting solids interact with one another in a variety of processes including thermal swelling, electrical conduction, electrochemical reactions, friction, and adhesion [7-9]. In energy storage and conversion applications the effective mechanical and electrical properties of granular electrode structures can be connected to microstructural characteristics [2, 10]. However, the interfacial properties in the existing modelling approaches are usually simplified [11].

Energy losses at interfaces are usually associated with ohmic heating (also known as Joule heating) due to the passage of an electrical current through contacting surfaces. In the context of energy management, improved electrical contacts play a prominent role in mitigating energy losses in battery assemblies. The energy loss due to the electrical contact resistance (ECR) at interfaces between electrode layers and at contacts between electrodes and current-collectors can be as high as $20 \%$ of the total energy flow of the batteries under normal operating conditions [12, 13]. The effects of the mechanical properties and surface roughness of electrical contacts on the performance of electrical connectors are of great importance in terms of potential drop and heat accumulation in contact zones [14, 15]. A significant increase in ECR can be caused by interfacial resistance due to the inevitable presence of resistive surface films, including corrosion deposits, fracture debris, oxide and hydrated layers at electrical contacts, resulting in excessive ohmic heating. In extreme cases, the heat can bring about system failure through sparks, fire and even melting of system components $[12,16,17]$.

The stress dependence of ECR at rough surfaces can be associated with the varying true interfacial contact area during system operation. However, the direct quantitative evaluation of real interfacial contact area between bodies through either experimental measurements or numerical simulations remains highly challenging due to the complex multi-scale morphologies exhibited by rough surface structures [1820]. Significant difficulties remain in relating interfacial electro-mechanical properties to surface structure descriptors.

Employing electrical measurement, nanoscale mechanical testing and surface morphology characterization, we investigated interfacial normal contact stiffness and electrical conduction behaviour at rough interfaces with random multiscale morphologies. First, we conducted contact stiffness measurements using flat-tipped diamond nanoindentation tests on a set of rough surfaces. Then, we examined the evolution of electrical conduction with varying compressive loads. Based on these results, discussions are extended to the relationship between electrical contact conductance and contact stiffness. This study demonstrates the importance of a multi-physics understanding of the origins of the electromechanical behaviour at interfaces in order to improve the reliability and performance of electrical contacts in energy systems. 


\section{Theoretical background}

Compared with the apparent or nominal contact area, the true area of contact at an interface is considerably smaller due to the existence of surface roughness. As shown in Fig. 1, when an electric current is conducted between two contacting solids, the restricted contact area, which depends on the size and spatial distribution of contacting asperities, causes additional constriction resistance (known as the electrical contact resistance, ECR) [21]. In addition to the constriction resistance resulting from the limited areas of true contact at an interface, ECR is also affected by the existence of resistive surface films, such as oxide layers [8]. Theories of ECR have since been further developed to include the effects of elastic-plastic deformation of the contacting asperities due to applied forces, multi-scale surface topography, size effects, and the contribution of insulating films between contacting bodies [18, 22-25].

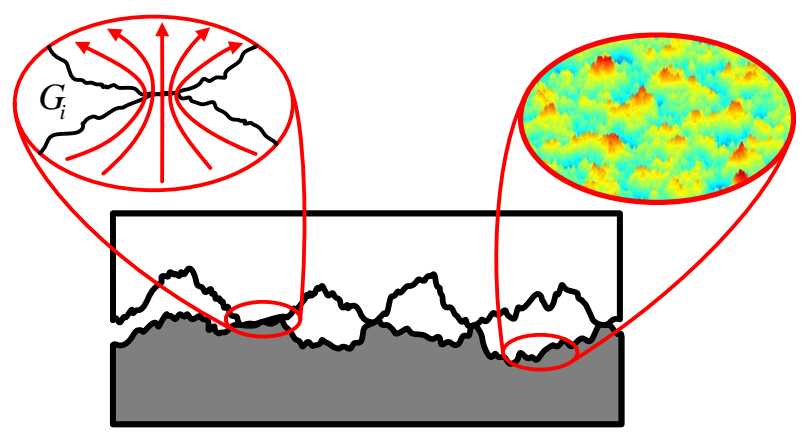

Fig. 1. Schematics of electrical conduction through a rough interface exhibiting multi-scale surface features.

Current flowing through rough interfaces is scattered across a large number of microcontacts of various geometries, which are often assumed to be circular in theoretical treatments $[19,26]$. The constriction resistance due to the convergence and divergence of current flow at a single contact is represented in Fig. 1. The resistance of a single contact is dictated by the dominant electronic transport mechanism, which depends on the contact area and structure. When the radius of the micro-contact, $r$, is comparable or smaller than the average electron mean free path, $\lambda$, the constriction resistance is dominated by the Sharvin mechanism, in which electrons travel ballistically across the microcontacts. The resistance of a contact with area, $a$, is given by [27].

$$
R_{S}=\frac{\lambda\left(\rho_{1}+\rho_{2}\right)}{2 a}
$$


where $\rho_{1}$ and $\rho_{2}$ are the specific resistivities of the contacting surfaces. On the other hand, when $r>\lambda$, the electron transport through the contact can be treated classically (Holm contact). The resistance can be expressed in the following form [8]

$$
R_{H}=\frac{\sqrt{\pi}\left(\rho_{1}+\rho_{2}\right)}{4 \sqrt{a}} .
$$

The total electrical conductivity, $G_{c}$, of an interface is assumed to be the sum of individual conductivity $G_{i}=R_{i}^{-1}$ at microcontacts, corresponding to the restriction resistances in parallel:

$$
G_{c}=\sum G_{i}
$$

In the case of rough surfaces with multiple contacting asperities, there is a distribution in the size of the contact area. The Sharvin and Holm expressions should therefore be considered as limiting cases.

\section{Surface preparation and characterisation}

Round disks, with a diameter of $25 \mathrm{~mm}$, made of aluminium alloy 5005 were used to fabricate specimens for both the measurement of the interfacial contact stiffness and ECR. For each individual sample, both the top and bottom surfaces were subjected to the same treatment using standard polishing and sand blasting procedures. The average diameters of the two selected groups of glass beads used in blasting treatments were $50 \mu \mathrm{m}$ and 300
In general, the area of contacts used in Eqs. (1) and (2), and therefore the contact resistance, depends on the applied pressure. Using theoretical and numerical approaches $[18,22,28,29]$, power-law type semiempirical correlations between the contact resistance and the normal pressure have been proposed for rough interfaces. In particular, previous theoretical studies found the contact conductance to be linearly proportional to the incremental stiffness [18, 22].

It should be noted that many mechanisms of surface structure evolution have been observed during electrical conduction through rough interfaces, including dielectric breakdown of oxide layers, localised current-induced welding, chemical disorder arising with random composition and oxidation processes in corrosive environments, and surface diffusion [30, 31]. These phenomena are outside the scope of this paper.

$\mu \mathrm{m}$. The sand blasting process was conducted for one minute, a duration which was sufficient to yield homogeneously and isotropically modified surface features. The sample surfaces were fabricated in such a way that each set of surfaces exhibited a distinct combination of surface roughness indicators, namely root mean squared (RMS) roughness and fractal dimension. Fig. 2 shows scanning electron microscope (SEM) images and typical surface profiles of the different surface types used in this work. Based on the threedimensional digitised topographies 
obtained by optical surface profilometry (NanoMap 1000WLI), the mean values of RMS roughness, fractal dimension and RMS slope with standard deviations over ten scans on different samples were calculated, as shown in Table 1. These values are found to be comparable with descriptors of naturally occurring surfaces [32]. Values of RMS roughness were calculated as the RMS average of the profile height from the scanning. In the digitised scanning, the slopes of triangular units formed by three adjacent pixels are used to calculate the RMS slope, which is commonly chosen as a higher order surface descriptor [33, 34]. The scaled triangulation method [34] was used for the calculation of fractal dimension values. It was found that the smaller the particles used to modify the surfaces the larger the fractal dimension was. The fractal dimension, a cross-scale surface descriptor that incorporates localised and macroscopic surface information provides an effective means for modelling engineering surfaces with random self-affine multi-scale properties in the characterisation of surfaces and particles [35]. The advantage of using surface fractality as a cross-scale surface descriptor stems in part from the tendency of first order descriptors (e.g., maximum height or mean roughness of the surface) to be dominated by highest scale features, while secondary descriptors (e.g., slope) tend to be dominated by finest scale surface characteristics $[33,36]$.
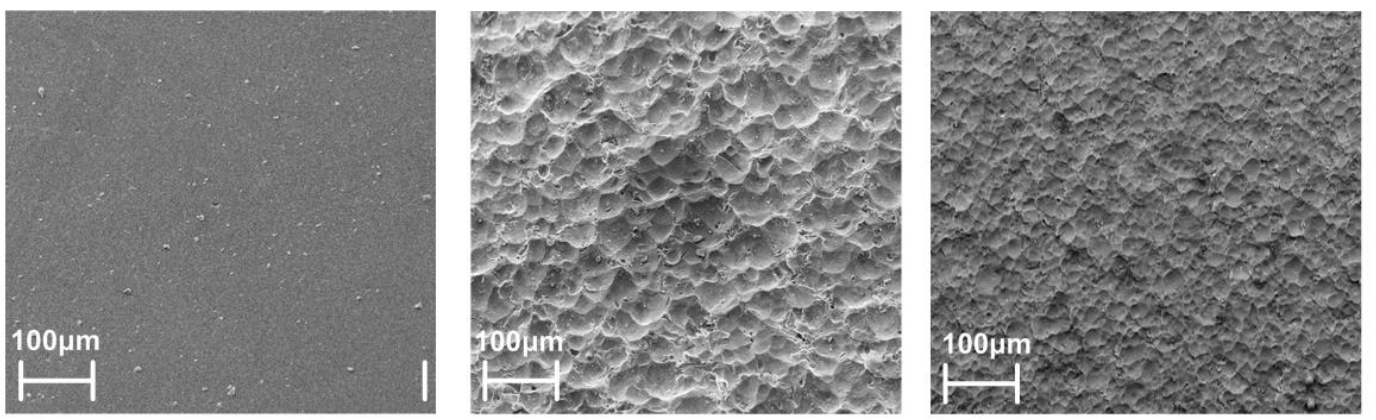

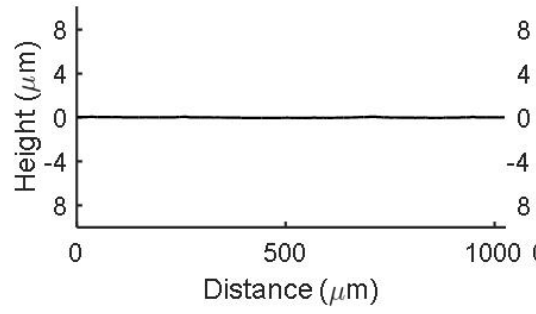

(a)

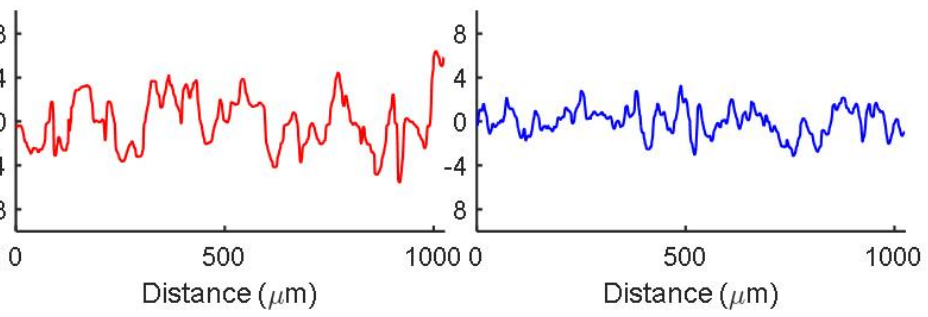

(b)

(c)

Fig. 2. SEM images and typical surface profiles of aluminium samples subjected to different surface treatments: (a) polished, S1; (b) sand blasted with $300 \mu \mathrm{m}$-sized glass beads, S2; (c) sand blasted with $50 \mu \mathrm{m}$-sized glass beads, S3. 


\section{Contact stiffness at rough surfaces}

The surface contact stiffness of aluminium samples with different surface morphology was assessed using nanoindentation (Agilent G200) with three flat indenter tips of different diameters of $54.1 \mu \mathrm{m}, 108.7$ $\mu \mathrm{m}$, and $502.6 \mu \mathrm{m}$ (FLT-D050, FLT-D100, and FLT-D500, respectively, SYNTONMDP, Switzerland). The reason for choosing flat tips is that the apparent contact area under the tip does not change with respect to the indentation depth, which is not the case for spherical or Berkovich tips. When the flat indenter tip first comes into contact with the testing sample, the actual contact area is only a small fraction of the nominal contact area. The asperities of the sample surface at contact regions are then squeezed against the flat tip as indentation progresses as is shown in Fig 3. In order to evaluate only the elastic responses, partial unloading tests were successively performed at ten intervals by decreasing the applied load by $10 \%$ each time. The loading level of each subsequent unloading stage is twice that of the previous unloading stage, with a maximum load of $500 \mathrm{mN}$ during the last unloading step.

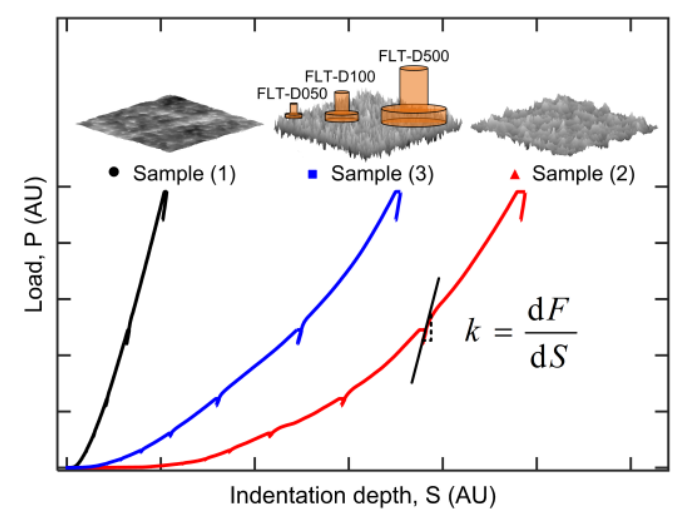

Fig. 3. Typical loading-displacement curves of nanoindentation tests on three types of surfaces. Ten partial unloading tests were carried out to isolate elastic contributions to contact stiffness under different loading levels. The three flat indenter tips used in the experiments (FLT-D050, FLT-D100, FLT-D500) are also illustrated for comparison.

Mean stiffness values were obtained by averaging data of ten indentation tests at different locations for each surface type. The unloading stiffness is here defined as the initial slope of the unloading curve, $k=$ $\mathrm{d} F / \mathrm{d} S$, where $F$ designates the normal force and $S$ is the indentation depth. Subsequently, the reduced elastic modulus
$E_{r}$ was derived from the measured unloading stiffness as

$$
k=\beta \frac{2}{\sqrt{\pi}} E_{r} \sqrt{A},
$$

where $A$ is the apparent contact area of the indenter tip and $\beta$ is a geometrical constant, taken as unity for a flat punch [37]. Eq. (4) is a fundamental equation for assessing the 
elastic properties in nanoidentation tests. The reduced modulus depends on the elastic properties of both the tested specimen and the indenter tip:

$$
\frac{1}{E_{r}}=\frac{1-v_{c}^{2}}{E_{c}}+\frac{1-v_{i}^{2}}{E_{i}},
$$

where $v_{i}$ and $v_{c}$ represent the Poisson's ratios of the indenter tip material and the tested specimen respectively. For the diamond indenter tips used in this research, $E_{i}$ and $v_{i}$ are typically $1140 \mathrm{GPa}$ and 0.07 , respectively. Equations (4) and (5) allow the estimation of $E_{c}$ from measured values of $A$ and $k$, while for $v_{c}$ we simply use the Poisson's ratio $v^{*}$ of bulk aluminium $\left(v_{c}=\right.$ $\left.v^{*}=0.3\right)$.

By using different sized flat tips, the stress range extends over several orders of magnitude. With the same maximum force $(500 \mathrm{mN})$ provided by the nanoindentor, the maximum stress produced with FLT-D050 was around 100 times larger than that produced with FLT-D500. The stress provided by all the three indenter tips ranged from $0.005 \mathrm{MPa}$ to $200 \mathrm{MPa}$, spanning five orders of magnitude. The contact stiffness measured over this range of applied stresses varies approximately from $0.01 \mathrm{GPa}$ to $55 \mathrm{GPa}$.

Fig. 4 shows the evolution of the contact stiffness with the applied force for the different surfaces. Here, we normalised the contact elastic modulus $E_{c}$ by the Young's modulus of aluminium alloy $5005, E^{*}=$
69.5 $\mathrm{GPa}$. The force is normalised by $E^{*} A$, where $A$ is the projected area of the corresponding tip. The measured contact stiffness increases with the loading force, for all tested samples. At the same applied stress level, the surfaces after sand blasting treatment (samples 2 and 3) show a smaller value of contact stiffness with respect to that of the polished surface (sample 1). The surface blasted with glass beads of $50 \mu \mathrm{m}$ diameter (sample 3) presents the lowest contact stiffness of all the three types of surfaces.

We express here the power-law relation of the contact stiffness with the applied normal force

$$
E_{c} \propto(F)^{\alpha_{E}},
$$

where $\alpha_{E}$ is the exponent of the power-law function $[38,39]$. It should be noted that the fitting curves are achieved excluding the contributions from the measured stiffness under stress levels higher than $100 \mathrm{MPa}$, where the surface shows an apparent yield phenomenon. For all the three surface types, the value of the exponent $\alpha_{E}$ varies from 0.4626 to 0.6048 (in Table 1), changing as the fractal dimension increases. In comparison, the typical value in cases of Hertzian contact of two elastic spheres is $1 / 3$, as shown in section 6 . The power-law relationship found here experimentally is in good agreement with previous theoretical predictions on a quantitative basis $[18,38$, 39]. 


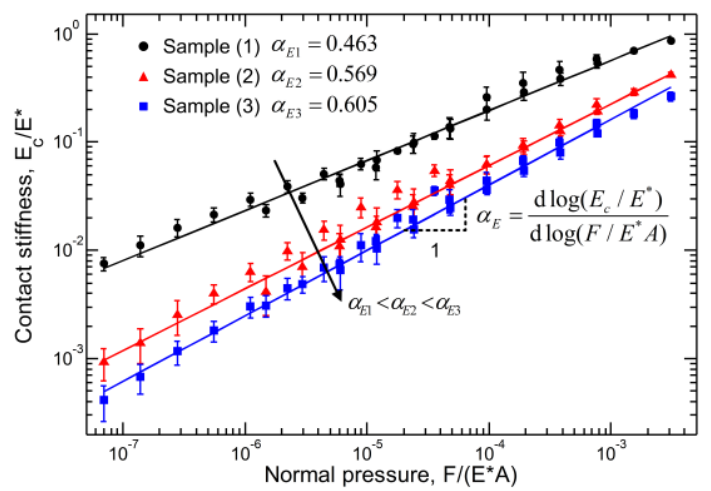

Fig. 4. Curve fitting for the normalised stiffness, $E_{c} / E^{*}$, and the normalised applied force, $F /\left(E^{*} A\right)$, for three tested surfaces, with $E^{*}$ being the Young's modulus of the tested material, and $A$ the apparent contact area.

\section{Electrical conductance at rough surfaces}

For each surface type, interfacial electrical conductance was measured for stacks of eleven disks, giving ten rough-to-rough interfaces. Analysis was achieved by means of a source/measurement unit (SMU B2900A, Agilent) across a range of applied compressive loading forces. In this experimental setting, we measured the resistance created by ten interfaces instead of a single interface, aiming to achieve a higher precision, larger linear range and better robustness against the measurement noises from the connecting wires, loading device and measurement unit. Using multiple interfaces further reduces experimental errors arising from inhomogeneity in surface treatment processes.

Prior to the measurement of forcedependent resistance, we performed resistance creep tests and sweeping current tests to select the most appropriate testing current and time to minimize influences on the measurement from the applied current. Full procedures and results have been previously published in greater detail [17]. The applied sweep current test consisted of two phases: a "loading" phase (P1) with current increasing logarithmically from $0.0001 \mathrm{~A}$ to $1.5 \mathrm{~A}$, followed by an "unloading" phase (P2) with current decreasing logarithmically from 1.5A back to $0.0001 \mathrm{~A}$. Both phases were conducted under conditions of constant normal load. During the sweeping loops, the voltage was recorded at a frequency of $2 \mathrm{kHz}$. The twophase sweeping process was completed within 0.2 seconds in order to avoid significant time dependant resistance degradation.

Fig. 5 shows the typical resistance-current characteristics for polished samples obtained from sweeping current tests. Each individual loop corresponds to a distinct load. The five loops shown demonstrate similar trends known as the Branly effect $[30,31]$, i.e., the measured resistance 
begins to drop irreversibly after the testing current reaches a certain value. The process is featured by voltage creep, hysteresis loops, and voltage saturation effects [31, 40]. The corresponding threshold current values for loops (1-3) are approximately $150 \mathrm{~mA}, 200 \mathrm{~mA}$ and $400 \mathrm{~mA}$, respectively, and the value seems to be positively correlated with the applied normal load. However, the Branly effect tends to be harder to capture at sufficiently high stress levels, shown in loops (4-5). For all five loops, when the testing current is higher than approximately $5 \mathrm{~mA}$ and lower than the threshold current values, the measured resistances remain stable at two plateaus in both $\mathrm{P} 1$ and $\mathrm{P} 2$, and can therefore be defined as ohmic resistance (the testing current is directly proportional to the measured voltage). At low testing currents (lower than $1 \mathrm{~mA}$ ), the measured resistance exhibits instability with the prominent measurement noises. The measured resistance obtained from subsequent sweeping tests will follow the path of the unloading phase (shown in the dashed lines in Fig. 5) [17].

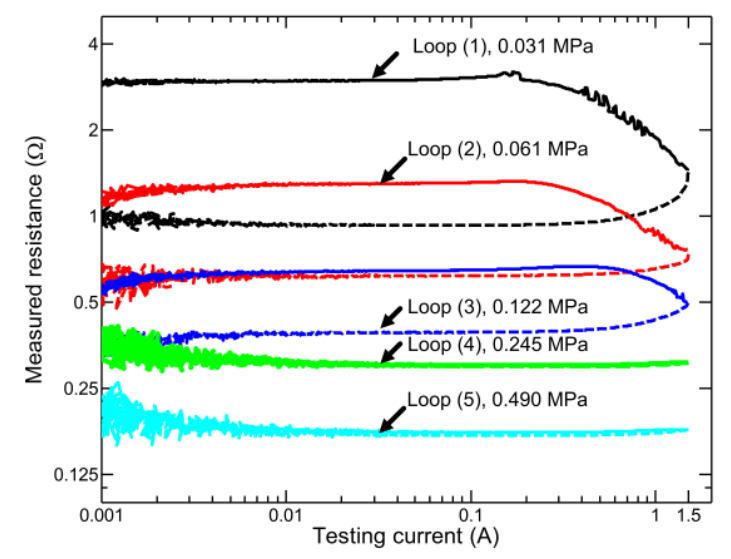

Fig. 5. Typical measured results for polished samples using current sweep under various stresses (0.031 MPa, 0.061 MPa, 0.122 MPa, 0.245 MPa and 0.490 MPa, corresponding to loops 1-5, respectively) with solid lines representing the first phase (P1) and the dashed lines showing the second phase (P2).

The experimental results in Fig. 5 indicate that both mechanical loading and electrical current alter the surface morphology and broaden the gap in measured resistance between P1 and P2. The contacting asperities can be regarded as a resistor network changing with the applied current, mechanical load, and measurement time. At the interfaces, the electrical current results in the physical and chemical modification of sample surfaces, which involves many processes, including the rupture of the oxide layer due to compression, and the localized heating induced by current. A high level of applied stress leads to better stability and repeatability of ECR measurements [17, 31]. 
Based on the performed sweeping current tests the electrical conductance resistance was measured under various stresses, for samples exhibiting different surface morphologies. For each type of sample, five series of tests were conducted and the resistances were evaluated at 16 different stress levels from 0.020 MPa to 8.936 MPa. The measured time was 0.01 second for each individual data point, in order to avoid significant effects arising from ohmic heating and associated time dependant resistance degradation. The testing current was set at $10 \mathrm{~mA}$, where all the three types of samples display an ohmic behaviour under varying electrical and mechanical loads. The interfacial electrical contact conductance was subsequently calculated through $G_{c}=1 /\left(R_{c}-R_{0}\right)$, where $R_{0}$ ( $0.06 \Omega$ ) is the combined resistance of the bulk material of identical size as the disk stack $(\sim 2.53 \mu \Omega)$, wires and connections used in the experimental setting.

As shown in Fig. 6, the measured conductance of disk stacks increases considerably with pressure, converging to a value close to the bulk conductance of the material. For given stress levels ( $\leq 4 \mathrm{MPa})$, samples blasted with $50 \mu \mathrm{m}$ sized glass beads (S3) usually present the lowest conductance among all the three types of samples. At low levels of applied stress (less than $0.5 \mathrm{MPa}$ ), the conductance is spread across a wider range. Similar to Eq. (6), we use a power-law function to express the correlation of the conductance with applied normal load as

$$
G_{C} \propto(F)^{\alpha_{G}}
$$

By fitting the conductance/pressure curves from $0.031 \mathrm{MPa}$ to $3.973 \mathrm{MPa}$, the power law exponent $\alpha_{G}$ is found to be 0.816 , 1.026 and 1.494 respectively for polished surfaces (S1), surface blasted with $300 \mu \mathrm{m}$ particles (S2) and those treated with $50 \mu \mathrm{m}$ particles (S3). The exponent values increase with the fractal dimension, shown in Table 1. Moreover, for all the three types of surfaces as shown in Fig. 6, the electrical conductance reaches a plateau under higher stresses, with the plateau value correlating to the RMS roughness. In the lower stress regime, the experimental data no longer seems to follow the power law. 


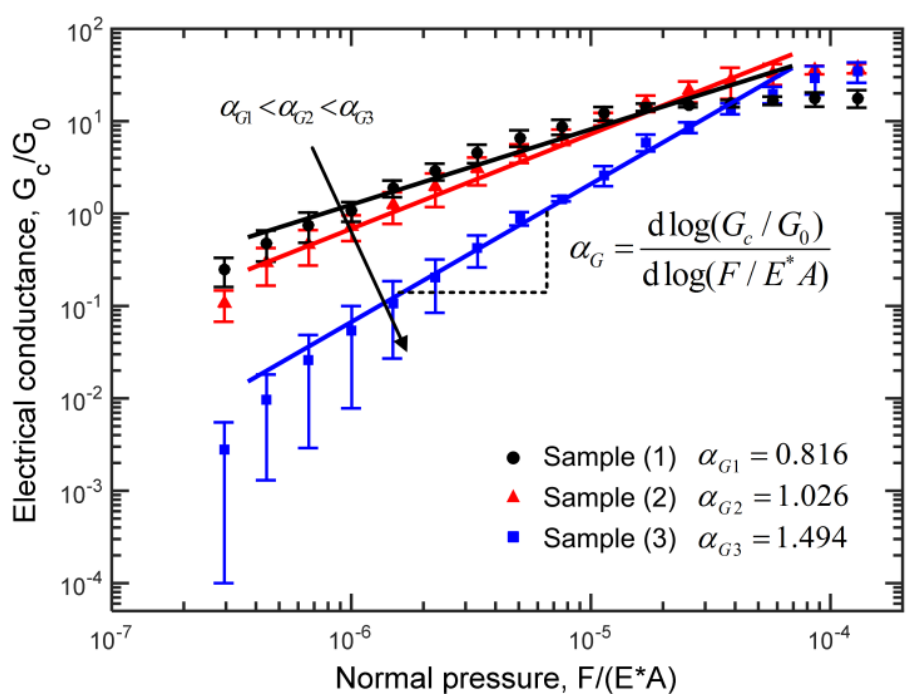

Fig. 6. Stress-dependent electrical conductance of different surfaces under various loading levels with a testing current of $10 \mathrm{~mA}$, with $E^{*}$ being the value of the Young's Modulus of the tested material, $A$ the projected area of the tested samples, and $G_{0}$ being set to $1 \Omega^{-1}$. The curve fitting were conducted for loading levels in a range of [0.031 MPa, 3.973 MPa].

\section{Discussion}

The key experimental results for contact stiffness and electrical conductance, measured for three types of rough surfaces, are summarised in Table 1. Both the contact stiffness and electrical conductance increase with the applied force, exhibiting power law behaviours with exponents $\alpha_{E}$ and $\alpha_{G}$, respectively. These exponents vary with the surface roughness and increase with the fractal dimension. In contrast, no evident correlation between the RMS roughness value and the exponent was found. This suggests that the correlation between contact stiffness, electrical conductance and applied force is dominated by fine scale surface characteristics.

We rationalize the experimental findings by developing the following scaling arguments. Both the contact stiffness and conductance primarily depend on the true contact area $A_{c}$, which evolves during mechanical loading and cannot be determined in a direct way based on the considered measurement methods. As a workaround, we estimate the true contact area based on the following expression for the incremental stiffness:

$$
k=\beta^{\prime} \frac{2}{\sqrt{\pi}} E_{r}^{\prime} \sqrt{A_{c}},
$$

where $E_{r}^{\prime}$ is the (constant) reduced elastic modulus calculated for the bulk elastic properties of the tested material and indenter: $\quad E^{\prime}{ }_{r}=\left(\left(1-v^{* 2}\right) / E^{*}+(1-\right.$ $\left.\left.v_{i}^{2}\right) / E_{i}\right)^{-1}$, and $\beta^{\prime}$ is a geometric factor of the order of unity. By writing Eq. (8), we assume that the effect of surface roughness on the measured incremental stiffness can be described by considering the true contact area $A_{c}$ (rather than the project contact area 
$A)$ and bulk material properties in the fundamental Eq (4). By comparing Eqs (4) and (8), and considering that $E_{i} \gg E^{*}>$ $E_{c}$, we obtain the following scaling relation:

$$
E_{c} / E^{*} \propto \beta^{\prime} \sqrt{A_{c} / A} .
$$

Next, we consider the true contact area to be the sum of $n$ individual contact areas, with average $a=A_{c} / n$. Here, individual asperities are assumed not to interact with one another during deformation. In order to relate the evolution of $a$ to the applied force, we first rely on a classical result of Hertzian contact theory. Representing a single contact by two spheres with radii $R_{1}$ and $R_{2}$ squeezed against each other, the contact area varies with the applied force according to

$$
a=\pi\left(\frac{3 R F}{4 E_{r}^{\prime}}\right)^{2 / 3}
$$

where $\quad R=\left(1 / R_{1}+1 / R_{2}\right)^{-1}$ is the equivalent radius of the two spheres, and the reduced modulus $E_{r}^{\prime}$ was introduced in Eq. (8). Eqs. (9) and (10) indicate that the contact stiffness is a power function of the load, with an exponent $1 / 3$. This simple scaling analysis is not consistent with our experimental findings for $\alpha_{E}$, which takes significantly higher values. However, the scaling analysis based on the Hertzian contact theory does not consider the changing number of contact asperities, $n$, for the increasing load. Furthermore, at the rough interface, the contact areas are not uniformly distributed [41], and interactions between asperities can exhibit complex deformation mechanisms, such as plastic deformation, adhesion, and friction.

On the other hand, introducing relation (10) into Eqs. (1) and (2) for the Holm and Sharvin resistance at a single contact, one finds that:

$$
G_{H}=\frac{4}{\tilde{\rho}}\left(\frac{3 R F}{4 E_{r}^{\prime}}\right)^{1 / 3}, G_{S}=\frac{2 \pi}{\lambda \tilde{\rho}}\left(\frac{3 R F}{4 E_{r}^{\prime}}\right)^{2 / 3},
$$

where $\tilde{\rho}=\left(\rho_{1}+\rho_{2}\right)$. Combining (11) with (3), we find that the total conductance of the rough surface, $G_{c}$, approximately scales with the force following a power law with the exponent ranging from $1 / 3$ (Holm) to 2/3 (Sharvin), depending on the dominant conduction mechanisms at individual contacts.

We further consider the contact model for a conical punch [42], where the contact area, $a$, is found to be linearly proportional to the applied force, $F$. With the same analysis as above, an exponent $\alpha_{E}=0.5$ can be derived for the contact stiffness, and an exponent $\alpha_{G}=0.5 \sim 1$ for the electrical conductance. This provides a better representation for the exponents of contact stiffness and electrical conductance as compared to the prediction by the Hertzian solution. Again, the exponents derived from this simple scaling analysis are lower than the experimental values of $\alpha_{G}$. This may also be due to the fact that the scaling neglects the increase in number of contacting points under increasing compression.

Despite these discrepancies, it is interesting to consider the ratio of the exponents for 
contact stiffness and electrical conductance, $\alpha_{G} / \alpha_{E}$. This ratio characterizes the power law relation between the conductance and contact stiffness, with $G_{C} \propto\left(E_{c}\right)^{\alpha_{G} / \alpha_{E}}$. According to the scaling analysis, this ratio ranges from 1 (Holm mechanism) to 2 (Sharvin mechanism). Experimentally, an approximate value of 2 was found for loading levels in a range of $F /\left(E^{*} A\right) \in$ $\left[5 \times 10^{-7}, 5 \times 10^{-5}\right]$. For sample 3 , similar fitting in the low load region gives a higher value of $\alpha_{G}$, and hence a higher value of the ratio $\alpha_{G} / \alpha_{E}$, suggesting a larger proportion of Sharvin-type contacts at low loads. Similar observations seem to hold as well for samples 1 and 2, but the transition takes place at even lower loads. As the load increases, new asperities come into contact, the contacting points enlarge and small microcontacts merge forming large contacts, resulting in better conduction. The dominant conduction mechanism transitions from a Sharvin-type to a Holm-type with the exponent ratio decreasing from 2 to 1 . Under sufficiently high forces, and hence high contact areas, the electric and mechanical properties converge to those of the bulk material, as expected.

The ratio $\alpha_{G} / \alpha_{E}$ also tends to increase with the fractal dimension. A surface with a higher fractal dimension demonstrates Sharvin dominated conductance $\left(\alpha_{G} /\right.$ $\left.\alpha_{E} \sim 2\right)$, while a less fractal surface presents combined Sharvin and Holm-type conductance $\left(\alpha_{G} / \alpha_{E}\right.$ between 1 and 2$)$.

Note that in the contact stiffness measurements, the flat indenter tips can be considered as rigid flat surfaces $\left(E_{i} \gg E^{*}\right)$, corresponding to a rough-to-flat contact problem. In comparison, our interfacial electrical resistance experiments involve rough-to-rough contacts. However, a scaling analysis based on rough-to-flat contact would yield identical exponents in the power law functions (10) and (11) [28, 33].

Table 1. Sample surface characteristics for different surface treatments

\begin{tabular}{ccccccc}
\hline $\begin{array}{c}\text { Sample } \\
\text { type }\end{array}$ & $\begin{array}{c}\text { RMS } \\
\text { roughness } / \mu \mathrm{m}\end{array}$ & $\begin{array}{c}\text { Fractal } \\
\text { dimension, } \mathrm{D}_{\mathrm{f}}\end{array}$ & RMS slope & $\begin{array}{c}\text { Contact } \\
\text { stiffness, } \alpha_{E}\end{array}$ & $\begin{array}{c}\text { Electrical } \\
\text { conductance, } \alpha_{G}\end{array}$ & $\begin{array}{c}\text { Exponent } \\
\text { ratio, } \alpha_{G} / \alpha_{E}\end{array}$ \\
\hline $\mathrm{S} 1$ & $0.057 \pm 0.005$ & $2.093 \pm 0.062$ & $0.009 \pm 0.001$ & $0.463 \pm 0.022$ & $0.816 \pm 0.081$ & $1.762 \pm 0.194$ \\
$\mathrm{~S} 2$ & $4.179 \pm 0.194$ & $2.551 \pm 0.022$ & $0.224 \pm 0.015$ & $0.569 \pm 0.029$ & $1.026 \pm 0.049$ & $1.803 \pm 0.126$ \\
$\mathrm{~S} 3$ & $2.970 \pm 0.276$ & $2.626 \pm 0.017$ & $0.202 \pm 0.010$ & $0.605 \pm 0.022$ & $1.494 \pm 0.134$ & $2.469 \pm 0.239$ \\
\hline
\end{tabular}

In the experiments, both contact stiffness and conductance may be affected by oxide layers at the sample surfaces. Aluminium alloys ubiquitously exhibit thin passivated hydrous and oxide layers arising from reaction with atmospheric oxygen and water. This nanoscale layer exhibits locally 
divergent mechanical properties in a region of thickness typically less than $10 \mathrm{~nm}$, which is significantly less than the depth of indentation performed in the current work. The influence of oxide layers is thus expected to be of limited significance in the present contact mechanics study. In the analysis of ECR behaviour, the oxide layer acts as an insulator. However, due to its limited thickness, the measured conductance is only sensitive to the presence of this layer at lower loads. For this reason large measurement uncertainties are evident at low loads with the magnitude of these fluctuations dependant on specimen surface structure, as shown in Fig. 6. Therefore in this study the effect of the oxide layer is minimal and does not interfere with the findings.

The observations made in this study can provide insights into the physical origin of the topological dependence of transport phenomena in energy materials applied in conversion, storage and generation systems. Parametric studies in to the performance of energy systems often yield unexpected behaviour arising from changes to the structure or processing of complex materials such as granular electrodes [2, 10]. The present work suggests that the structure and mechanics of interfaces in these systems may be in part a contributing factor to the observed processing dependence of performance.

\section{Conclusion}


scientific and technical assistance of the Australian Microscopy \& Microanalysis Research Facility at the Australian Centre for Microscopy \& Microanalysis at the University of Sydney.

\section{References:}

[1] S.W. Lee, H.-W. Lee, I. Ryu, W.D. Nix, H. Gao, Y. Cui, Kinetics and fracture resistance of lithiated silicon nanostructure pairs controlled by their mechanical interaction, Nature communications, 6 (2015).

[2] W. Bauer, D. Nötzel, V. Wenzel, H. Nirschl, Influence of dry mixing and distribution of conductive additives in cathodes for lithium ion batteries, Journal of Power Sources, 288 (2015) 359-367.

[3] M.S. Islam, C.A. Fisher, Lithium and sodium battery cathode materials: computational insights into voltage, diffusion and nanostructural properties, Chemical Society Reviews, 43 (2014) 185-204.

[4] F. Abdeljawad, B. Völker, R. Davis, R.M. McMeeking, M. Haataja, Connecting microstructural coarsening processes to electrochemical performance in solid oxide fuel cells: An integrated modeling approach, Journal of Power Sources, 250 (2014) 319331.

[5] M.A. De Brito, L.P. Sampaio, L.G. Junior, C. Canesin, Research on photovoltaics: review, trends and perspectives, Power Electronics Conference (COBEP), 2011 Brazilian, IEEE, 2011, pp. 531-537.

[6] L.E. Bell, Cooling, heating, generating power, and recovering waste heat with thermoelectric systems, Science, 321 (2008) 1457-1461.

[7] K.L. Johnson, K.L. Johnson, Contact mechanics, Cambridge University Press, 1987.

[8] R. Holm, Electric contacts, Springer, New York, 1967.

[9] F. Jin, X. Guo, Mechanics of axisymmetric adhesive contact of rough surfaces involving power-law graded materials, International Journal of Solids and Structures, 50 (2013) 3375-3386.

[10] J. Ott, B. Völker, Y. Gan, R.M. McMeeking, M. Kamlah, A micromechanical model for effective conductivity in granular electrode structures, Acta Mechanica Sinica, 29 (2013) 682-698.

[11] R. Xu, K. Zhao, Mechanical interactions regulated kinetics and morphology of composite electrodes in Li-ion batteries, Extreme Mechanics Letters, (2015).

[12] P. Taheri, S. Hsieh, M. Bahrami, Investigating electrical contact resistance losses in lithium-ion battery assemblies for hybrid and electric vehicles, Journal of Power Sources, 196 (2011) 6525-6533.

[13] U.S. Kim, C.B. Shin, C.-S. Kim, Effect of electrode configuration on the thermal behavior of a lithium-polymer battery, Journal of Power Sources, 180 (2008) 909-916. [14] L. Kogut, I. Etsion, Electrical conductivity and friction force estimation in compliant electrical connectors, Tribology Transactions, 43 (2000) 816-822.

[15] V. Srinivasan, C. Wang, Analysis of electrochemical and thermal behavior of Li-ion cells, Journal of The Electrochemical Society, 150 (2003) A98-A106.

[16] S. Dorbolo, A. Merlen, M. Creyssels, N. Vandewalle, B. Castaing, E. Falcon, Effects of electromagnetic waves on the electrical properties of contacts between grains, EPL (Europhysics Letters), 79 (2007) 54001.

[17] C. Zhai, D. Hanaor, G. Proust, Y. Gan, Stress-Dependent Electrical Contact Resistance at Fractal Rough Surfaces, Journal of Engineering Mechanics, (2015) B4015001. 
[18] R. Pohrt, V.L. Popov, Normal contact stiffness of elastic solids with fractal rough surfaces, Physical Review Letters, 108 (2012) 104301.

[19] G. Carbone, F. Bottiglione, Contact mechanics of rough surfaces: a comparison between theories, Meccanica, 46 (2011) 557-565.

[20] D.A. Hanaor, Y. Gan, I. Einav, Contact mechanics of fractal surfaces by spline assisted discretisation, International Journal of Solids and Structures, 59 (2015) 121131.

[21] J. Greenwood, Constriction resistance and the real area of contact, British Journal of Applied Physics, 17 (1966) 1621.

[22] J. Barber, Bounds on the electrical resistance between contacting elastic rough bodies, Proceedings of the Royal Society of London. Series A: Mathematical, Physical and Engineering Sciences, 459 (2003) 53-66.

[23] L. Kogut, K. Komvopoulos, Electrical contact resistance theory for conductive rough surfaces separated by a thin insulating film, Journal of applied physics, 95 (2004) 576585.

[24] A. Mikrajuddin, F.G. Shi, H. Kim, K. Okuyama, Size-dependent electrical constriction resistance for contacts of arbitrary size: from Sharvin to Holm limits, Materials Science in Semiconductor Processing, 2 (1999) 321-327.

[25] R.L. Jackson, E.R. Crandall, M.J. Bozack, Rough surface electrical contact resistance considering scale dependent properties and quantum effects, Journal of Applied Physics, 117 (2015) 195101.

[26] L. Kogut, R.L. Jackson, A comparison of contact modeling utilizing statistical and fractal approaches, Journal of tribology, 128 (2006) 213-217.

[27] Y.V. Sharvin, On the possible method for studying fermi surfaces, Zh. Eksperim. i Teor. Fiz., 48 (1965).

[28] L. Kogut, K. Komvopoulos, Electrical contact resistance theory for conductive rough surfaces, Journal of Applied Physics, 94 (2003) 3153-3162.

[29] M. Paggi, J. Barber, Contact conductance of rough surfaces composed of modified RMD patches, International Journal of Heat and Mass Transfer, 54 (2011) 4664-4672. [30] K. Bourbatache, M. Guessasma, E. Bellenger, V. Bourny, A. Tekaya, Discrete modelling of electrical transfer in multi-contact systems, Granular Matter, 14 (2012) 110.

[31] E. Falcon, B. Castaing, M. Creyssels, Nonlinear electrical conductivity in a 1D granular medium, The European Physical Journal B-Condensed Matter and Complex Systems, 38 (2004) 475-483.

[32] B. Persson, O. Albohr, U. Tartaglino, A. Volokitin, E. Tosatti, On the nature of surface roughness with application to contact mechanics, sealing, rubber friction and adhesion, Journal of Physics: Condensed Matter, 17 (2005) R1.

[33] A. Majumdar, B. Bhushan, Role of fractal geometry in roughness characterization and contact mechanics of surfaces, Journal of Tribology, 112 (1990) 205-216.

[34] C. Zhai, Y. Gan, D. Hanaor, G. Proust, D. Retraint, The Role of Surface Structure in Normal Contact Stiffness, Experimental Mechanics, 56 (2016) 359-368.

[35] N. Almqvist, Fractal analysis of scanning probe microscopy images, Surface Science, 355 (1996) 221-228.

[36] C. Zhai, Y. Gan, D. Hanaor, G. Proust, D. Retraint, The Role of Surface Structure in Normal Contact Stiffness, Experimental Mechanics, (2015) 1-10.

[37] W.C. Oliver, G.M. Pharr, Measurement of hardness and elastic modulus by instrumented indentation: Advances in understanding and refinements to methodology, Journal of Materials Research, 19 (2004) 3-20. 
Zhai, Chongpu, et al. "Interfacial electro-mechanical behaviour at rough

surfaces." Extreme Mechanics Letters 9 (2016): 422-429.

[38] R. Buzio, C. Boragno, F. Biscarini, F.B. De Mongeot, U. Valbusa, The contact mechanics of fractal surfaces, Nature Materials, 2 (2003) 233-236.

[39] S. Jiang, Y. Zheng, H. Zhu, A contact stiffness model of machined plane joint based on fractal theory, Journal of Tribology, 132 (2010) 011401.

[40] S. Dorbolo, M. Ausloos, N. Vandewalle, Reexamination of the Branly effect, Physical Review E, 67 (2003) 040302.

[41] B.B. Mandelbrot, D.E. Passoja, A.J. Paullay, Fractal character of fracture surfaces of metals, Nature, 308 (1984) 5961.

[42] I.N. Sneddon, The relation between load and penetration in the axisymmetric Boussinesq problem for a punch of arbitrary profile, International Journal of Engineering Science, 3 (1965) 47-57. 